\title{
Manejo endoscópico de quiste de duplicación esofágico: descripción de un caso
}

\section{A case of endoscopic management of an esophageal duplication cyst}

\author{
Andrés Mauricio Ricardo Ramírez, ${ }^{1}$ Édgar Julián Ferreira Bohórquez, ${ }^{2}$ Lázaro Antonio Arango Molano, ${ }^{3}$ Andrés Sánchez Gil. ${ }^{4}$
}

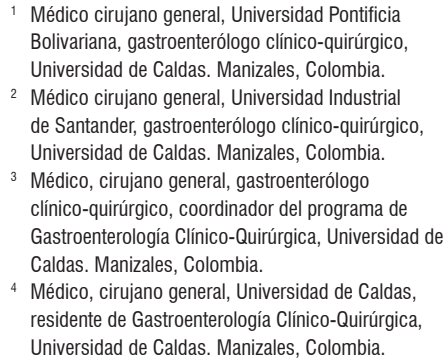

1 Médico cirujano general, Universidad Pontificia Bolivariana, gastroenterólogo clínico-quirúrgico, Universidad de Caldas. Manizales, Colombia. 2 Médico cirujano general, Universidad Industrial de Santander, gastroenterólogo clínico-quirúrgico Universidad de Caldas. Manizales, Colombia.

3 Médico, cirujano general, gastroenterólogo clínico-quirúrgico, coordinador del programa de Gastroenterología Clínico-Quirúrgica, Universidad de Caldas. Manizales, Colombia.

${ }^{4}$ Médico, cirujano general, Universidad de Caldas, residente de Gastroenterología Clínico-Quirúrgica, Universidad de Caldas. Manizales, Colombia.

Fecha recibido: $22-08-17$ Fecha aceptado: 13-04-18

\begin{abstract}
Resumen
Los quistes de duplicación esofágica son una anomalía congénita poco frecuente. La mayoría de los casos son asintomáticos y su diagnóstico frecuentemente es un hallazgo incidental. La posibilidad de que se desarrollen complicaciones (hemorragia, infección, entre otras) y el temor a la posibilidad de transformación maligna hacen que el tratamiento de estas lesiones haya sido considerado quirúrgico en el pasado. Sin embargo, la extremadamente baja taza de malignización y la posibilidad de un desenlace inadecuado asociado con la cirugía hacen considerar otras alternativas, teniendo en cuenta la relación riesgo-beneficio del procedimiento quirúrgico. Se reporta el caso de una paciente de 56 años con diagnóstico endosonográfico de quiste de duplicación esofágica, que fue sometida a marsupialización endoscópica del quiste con una resolución completa de sus síntomas, sin complicaciones y con una reintegración inmediata a la vida diaria.

La endosonografía (EUS) ha sido ampliamente utilizada como una herramienta para la evaluación y el diagnóstico de los quistes de duplicación. Es el método diagnóstico de elección para investigar esta patología ya que puede distinguir entre lesiones sólidas y quísticas. La cuestión de si se debe realizar o no la aspiración con aspiración con aguja fina guiada por EUS (FNA) en una lesión sospechosa, en el caso de un quiste de duplicación, es controvertida ya que estas lesiones pueden infectarse con consecuencias significativas, aunque frecuentemente se requiere que la punción obtenga un diagnóstico definitivo que descarte lesiones ominosas.
\end{abstract}

\section{Palabras clave}

Quiste, duplicación, esófago, manejo endoscópico.

\section{Abstract}

Esophageal duplication cysts are rare congenital anomalies. Most cases are asymptomatic and their diagnoses are often incidental findings. In the past, surgery was considered to be the treatment for these lesions because of the possibility of complications such as hemorrhaging and infections as well as the fear of malignant transformation. However, the extremely low rate of malignancy and the possibility of an inadequate outcomes of surgery have made it necessary to consider other alternatives that taking both risks and benefits of the surgical procedure into account. We report the case of a 56-year-old patient with an endosonographic diagnosis of an esophageal duplication cyst who underwent endoscopic marsupialization of the cyst followed by complete resolution of symptoms, without complications, and with immediate reintegration into daily life.

Endosonography (EUS) has been widely used as a tool for evaluation and diagnosis of duplication cysts. It is the diagnostic method of choice for investigating this pathology since it can distinguish between solid and cystic lesions. The question of whether or not to perform EUS guided fine needle aspiration (FNA) of a lesion suspected of being a duplication cyst is controversial since these lesions can be infected with significant consequences. Nevertheless, EUS-FNA is often required to make a definitive diagnosis that rules out ominous lesions.

\section{Keywords}

Cyst, duplication, esophagus, endoscopic management. 


\section{INTRODUCCIÓN}

Los quistes de duplicación esofágica son alteraciones benignas del desarrollo embrionario temprano hacia la tercera o cuarta semanas de la gestación. Son relativamente infrecuentes con una incidencia estimada es de 1 por cada 8000 nacidos vivos y se han descrito 2 tipos: adyacentes al lumen (sin comunicación con la luz esofágica) y tubulares (que se comunican directamente al lumen). Frecuentemente, estos 2 tipos de duplicación se reportan como entidades diferentes.

Presentan 3 características distintivas: se encuentran contenidos en la pared esofágica, están cubiertos por las 2 capas musculares y se encuentran revestidos por epitelio escamoso o un revestimiento compatible con el que se encuentra en el esófago embrionario (1).

Hasta un tercio de estos quistes contienen mucosa gástrica, pancreática o mucosa que contiene placas de Peyer en forma heterotópica. Aproximadamente, el $80 \%$ no se comunica con la luz del esófago y se produce con mayor frecuencia en la cara lateral derecha del esófago debido a la dextrorrotación del estómago durante la embriogénesis (2). Estas lesiones pueden causar síntomas que se observan más frecuentemente en la infancia ( $80 \%$ de los casos), generados por la compresión de las estructuras adyacentes que más comúnmente causan disfagia (70\%), dolor epigástrico (20\%), dolor retroesternal (10\%) (2) y síntomas respiratorios como tos, estridor y sibilancias. La hematemesis es más rara y se presenta en pacientes con comunicación tubular.

En los pacientes mayores, suelen diagnosticarse en forma incidental durante la endoscopia superior o en imágenes radiológicas, y la aparición de síntomas es infrecuente luego de los 2 años de edad (3).

Se han descrito casos de transformación maligna; sin embargo, su prevalencia real es desconocida y solo se encuentran 2 casos publicados: el reporte de McGregor en 1976, que corresponde a un diagnóstico post mortem por causas infecciosas; y el reporte de Sigh del 2001, que corresponde a un paciente de 18 años con adecuada respuesta a la terapia con quimiorradiación $(4,5)$.

La apariencia endoscópica de los quistes de duplicación puede corresponder con la apariencia descrita en otras lesiones subepiteliales del esófago, con una protuberancia luminal asociada con una mucosa normal suprayacente sin irregularidades; y en caso de duplicaciones tubulares, puede verse como un divertículo de tamaño variable.

Los hallazgos endosonográficos corresponden con lesiones homogéneas anecoicas con márgenes regulares derivados de la submucosa (tercera capa). Su pared puede estar constituida de 3 a 5 capas y hay algunos casos en los que la imagen es más hipo que anecoica debido a la presencia de material ecogénico, que consiste en capas de detritos o de mucina.
El diagnóstico se basa en las características endoscópicas y endosonográficas; y en los casos en los que persiste la duda asociada con la presencia de imágenes de contenido hipoecoico en el quiste, la aspiración con punción con aguja fina guiada por EUS (FNA) se ha utilizado para esclarecer el diagnóstico y descartar malignidad; pero en lesiones que son claramente anecoicas, esta práctica no se recomienda por el riesgo presuntivo de infección $(6,7)$. El manejo de quistes asintomáticos puede ser expectante, aunque el potencial de complicaciones, incluida la transformación maligna, ha hecho que algunos autores recomienden la resección quirúrgica. Sin embargo, no existen estudios prospectivos que evalúen la historia natural de los quistes de duplicación, por lo que las recomendaciones a este respecto carecen de fuerza estadística.

Para los casos sintomáticos se han descrito tanto el manejo quirúrgico como el endoscópico. Se describió por primera vez en un reporte de caso de Will y colaboradores de 2005, en el que se presentó el caso de un paciente de 25 años a quien se le realizó una fenestración exitosa sobre el quiste para crear una comunicación con la luz esofágica (8). Igualmente, se describió la resección completa de la pared luminal del quiste en una paciente de 75 años sintomática, a quien se le realizó la resección con asa de polipectomía debido a la característica pseudopediculada de la lesión (9).

En la EUS, los quistes de duplicación esofágica aparecen frecuentemente como una masa hipoecoica homogénea periesofágica, como ya se mencionó. Sin embargo, cuando hay dudas acerca de la malignidad, una herramienta es la FNA en el diagnóstico de quistes de duplicación esofágica y quistes de duplicación en general, aunque es controvertida. Mientras que la punción-aspiración con aguja fina (PAAF) puede mostrar células escamosas u otros contenidos de quistes compatibles con un quiste de duplicación que puede ayudar en el diagnóstico, el riesgo de infectar el quiste es una preocupación importante, con algunos estudios que han mostrado una tasa de infección tan alta como del 14\%. La EUS-FNA se debe realizar si hay una preocupación por una lesión más grave o si la naturaleza de la lesión está en duda.

\section{CASO CLÍNICO}

Se presenta el caso clínico de una paciente de 56 años sin antecedentes médicos de importancia, quien desarrolló disfagia para sólidos de 2 meses de evolución, con pérdida de $4 \mathrm{~kg}$ de peso durante ese lapso, por lo que se indicó una endoscopia digestiva superior. Se encontró una lesión de $30 \mathrm{~mm}$ hacia el tercio inferior del esófago en la pared anterior derecha, de apariencia subepitelial, cubierta por una mucosa sin alteraciones (Figura 1). 

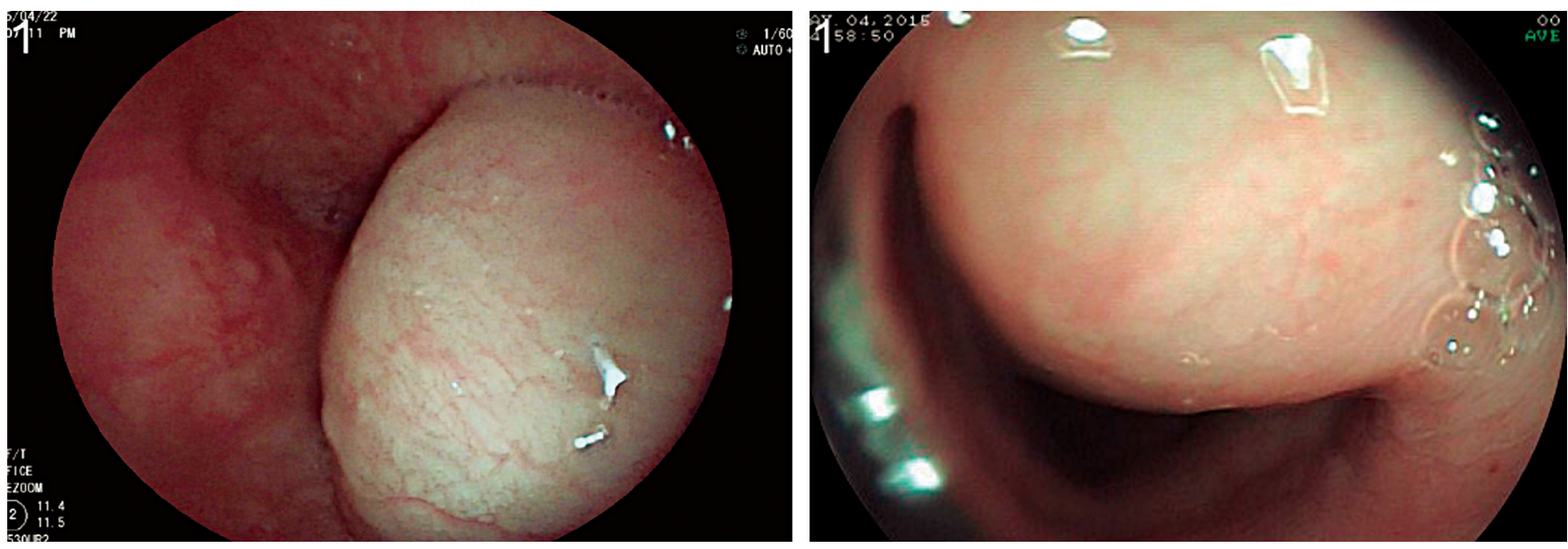

Figura 1. Visión endoscópica de la lesión subepitelial esofágica.

Ante el hallazgo, se decidió realizar la EUS radial esofágica en la que se encontró una lesión dependiente de la tercera capa esofágica, pero de características hipoecoicas más que anecoicas, por lo que se realizó una EUS-FNA con estudio citológico rápido. Se encontró abundante material mucoso que evidenció la presencia de células epiteliales y contenido de mucina, sin evidencia de cambios citopatológicos malignos (Figura 2).

Ante los hallazgos que apoyaron el diagnóstico de quiste de duplicación sin malignización, se procedió a realizar una marsupialización del quiste bajo anestesia general en una sección de la pared luminal del mismo con electrobisturí de punta endoscópico. Se realizó un corte de $20 \mathrm{~mm}$ sobre el cénit de la lesión luminal con disección por pasos hasta la submucosa; se logró el completo drenaje de la lesión y su inspección endoscópica completa, y se encontró una cavidad ciega, recubierta por un epitelio pálido, sin características de malignidad (Figura 3).

La paciente evolucionó satisfactoriamente con dolor retroesternal como el único síntoma posterior al procedi-
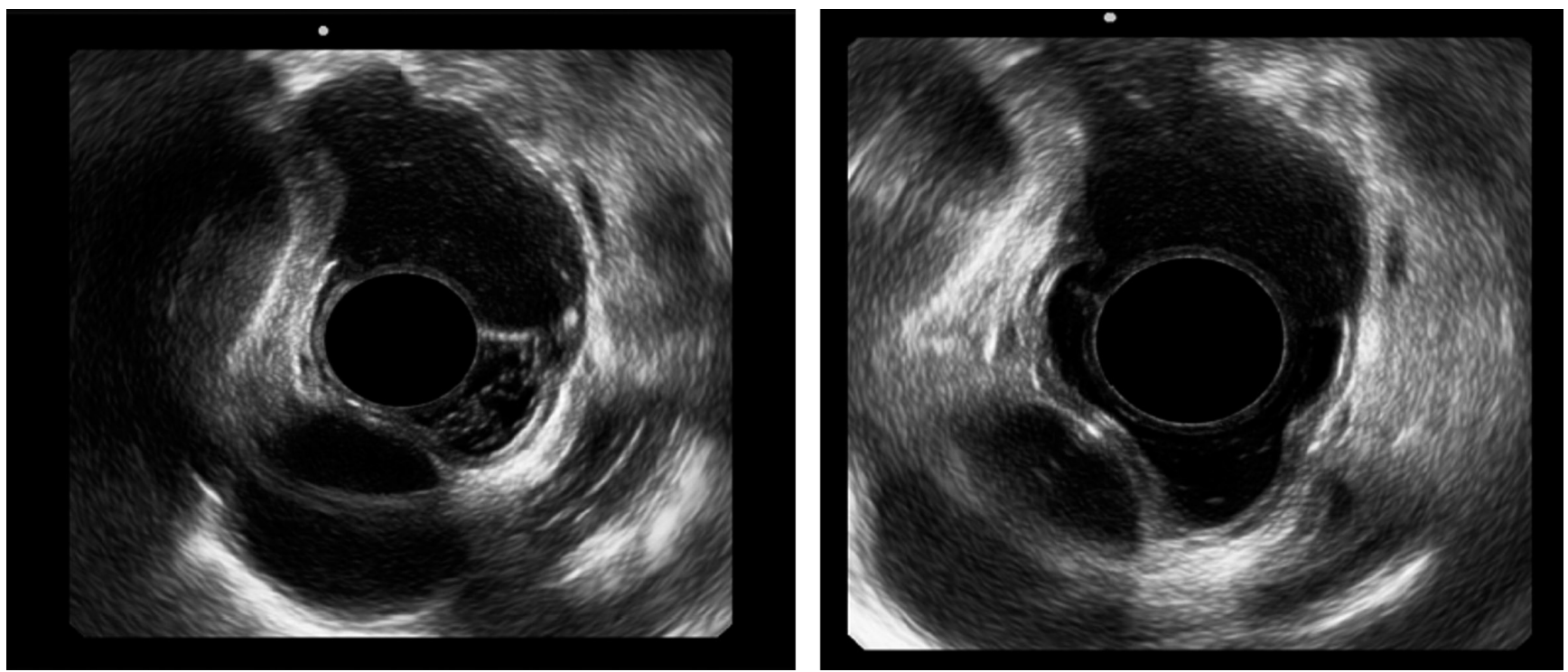

Figura 2. Endosonografía radial. Se observa una lesión subepitelial de la tercera capa esofágica, hipoecoica. 

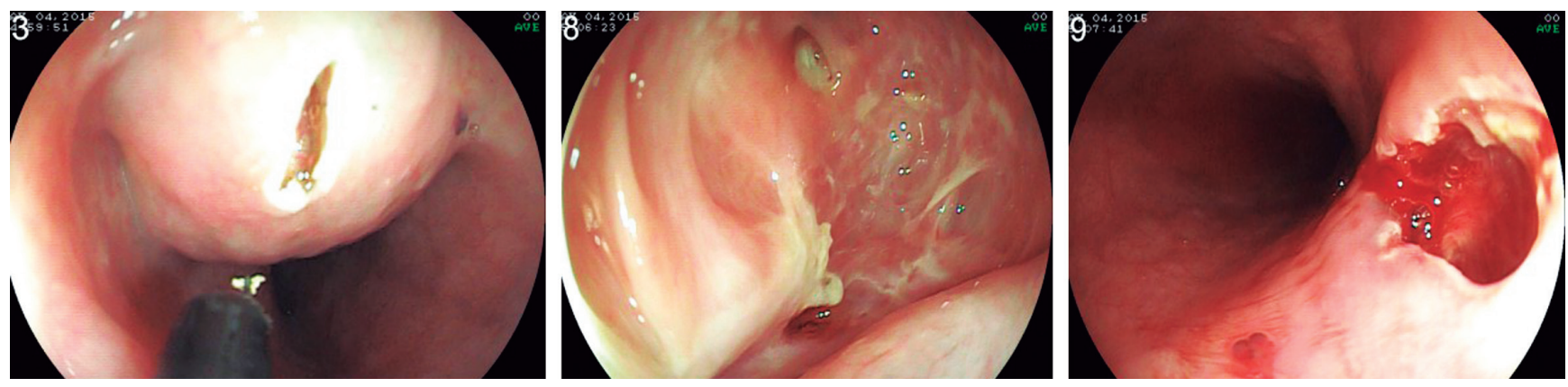

Figura 3. Incisión de la lesión. Se observa el quiste de duplicación por dentro y al final el orificio que queda a la luz del esófago.
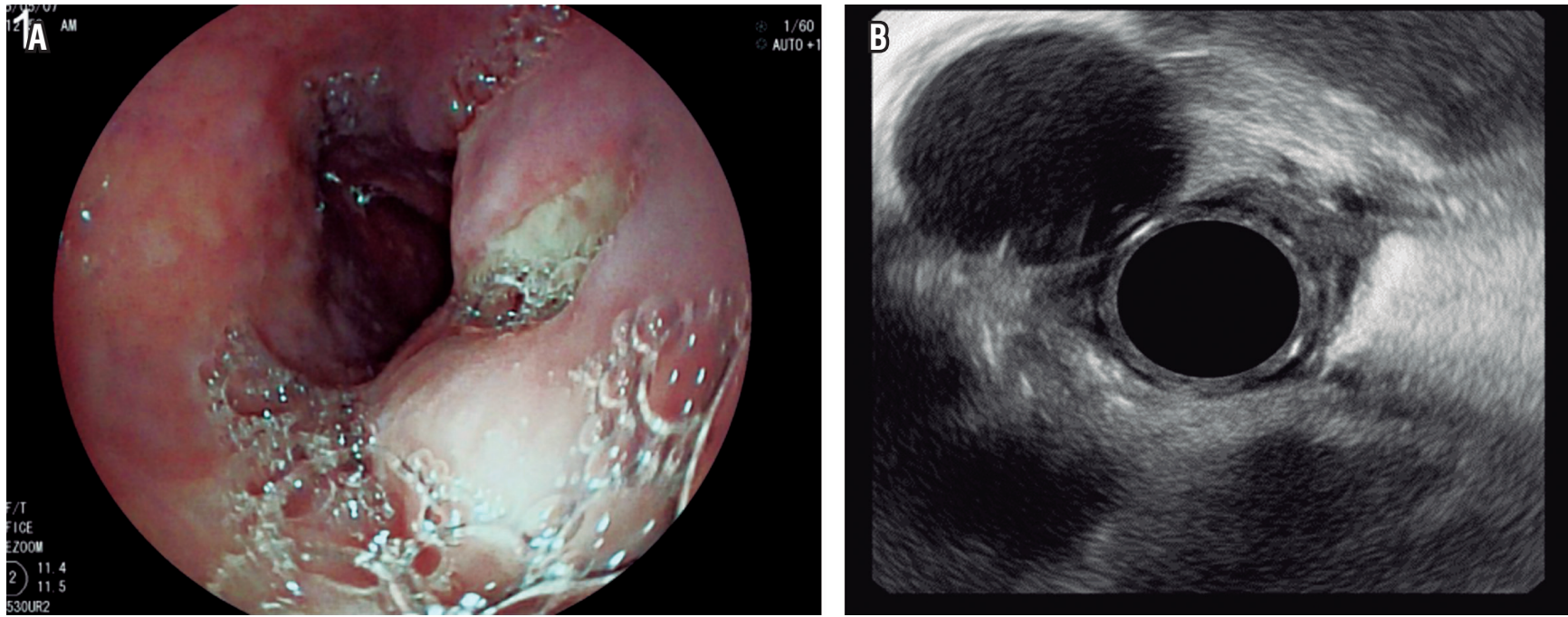

Figura 4. Control al cuarto día. A. Visión endoscópica que muestra el orificio externo a la luz del esófago. B. EUS. Aorta a las 11 del visor y el quiste ya reducido posdrenaje.

miento, que cedió con los analgésicos comunes en las primeras 24 horas. Se inició una prueba de tolerancia oral en ese momento, sin presentar disfagia residual.

El control endosonográfico se llevó a cabo hacia el cuarto día posterior al procedimiento y se encontró un colapso completo de la lesión (Figura 4 A), asociado con una comunicación total con la luz esofágica en ausencia de residuos alimentarios (Figura 4 B).

Debido a su excelente evolución y a no presentar síntomas residuales, la paciente fue dada de alta con protector de mucosa (sucralfato), pero sin requerimiento de analgésico en casa. Se realizó seguimiento telefónico a los 10 días en el que se evaluó la tolerancia a la vía oral y la paciente manifestó que habían desaparecido por completo los síntomas de disfagia que la llevaron a consultar en un principio.

\section{DISCUSIÓN}

Los quistes de duplicación esofágica son lesiones poco frecuentes asociadas con el desarrollo embrionario del intestino anterior. Su baja frecuencia hace que la historia natural de estas lesiones sea desconocida, aunque la búsqueda sistemática de complicaciones que impliquen su resección quirúrgica obligada como la presencia de malignidad no arrojan resultados que permitan un grado fuerte de recomendación de este tratamiento. Sin embargo, las complicaciones asociadas con la cirugía abierta o mínimamente invasiva pueden ser extrapoladas; de este modo se sabe que existe un riesgo entre el $15 \%$ y $37,5 \%$ de complicaciones, principalmente de tipo respiratorio, asociadas con la cirugía torácica; y en el caso de abordaje abdominal superior, el riesgo fluctúa entre 
$16 \%$ y $17 \%$ (10), lo que resulta altamente mórbido frente al manejo de una enfermedad benigna.

Igualmente, el desarrollo de técnicas endoscópicas carece de estudios de gran diseño metodológico, pero la menor invasividad y ausencia de alteración de la fisiología respiratoria los hace un método altamente atractivo para el manejo de los quistes de duplicación. La técnica de elección para el manejo endoscópico de los quistes de duplicación esofágica aún se encuentra en desarrollo y solo 2 reportes de caso han descrito resultados $(8,9)$. En el caso reportado por Will y colaboradores en 2005, se utilizó una técnica similar a la empleada en nuestro caso, pero la escasa amplitud de la ventana inicial $(10 \mathrm{~mm})$ obligó a una ampliación de la misma a las 6 semanas por recurrencia de los síntomas, por lo que manufacturar una ventana amplia se muestra indispensable para la marsupialización completa del quiste. En nuestro estudio se usó una amplitud intermedia determinada por el diámetro de la lesión y una técnica por pasos para maximizar la seguridad del procedimiento.

Una vez establecido el diagnóstico de quiste de duplicación, el tratamiento puede variar dependiendo de la presencia de los síntomas. En los pacientes sintomáticos, la resección quirúrgica es frecuentemente la opción para el alivio de los síntomas. En pacientes asintomáticos, la resección quirúrgica es controvertida: mientras que algunos autores defienden la resección debido a la posible degeneración maligna del quiste de duplicación, otros han apoyado la observación.

Puesto que ha habido informes de casos de quistes de duplicación estables en la vigilancia de EUS, este puede ser un método adecuado de seguimiento ambulatorio; $y$ la resección quirúrgica se puede considerar si el paciente desarrolla síntomas. En cualquier caso, el manejo quirúrgico frente al no quirúrgico de los quistes de duplicación asintomáticos probablemente siga siendo polémico hasta que se entienda más sobre el curso del tiempo y los factores de riesgo asociados con su degeneración maligna.

Hasta la fecha, no existen otros reportes que permitan obtener conclusiones extrapolables con respecto al manejo de los quistes de duplicación esofágica. Nuestro reporte de caso pretende plantear la seguridad y eficacia de los métodos endoscópicos en el tratamiento de estas lesiones y servir como base de futuros estudios que permitan dar luces respecto a esta patología infrecuente.

\section{REFERENCIAS}

1. Ildstad ST, Tollerud DJ, Weiss RG, et al. Duplications of the alimentary tract. Clinical characteristics, preferred treatment, and associated malformations. Ann Surg. 1988;208(2):184-9. https://doi.org/10.1097/00000658198808000-00009.

2. Arbona JL, Fazzi JG, Mayoral J. Congenital esophageal cysts: case report and review of literature. Am J Gastroenterol. 1984;79(3):177-82.

3. Harvell JD, Macho JR, Klein HZ. Isolated intra-abdominal esophageal cyst. Case report and review of the literature. Am J Surg Pathol. 1996;20(4):476-9. https://doi. org/10.1097/00000478-199604000-00011.

4. McGregor DH, Mills G, Boudet RA. Intramural squamous cell carcinoma of the esophagus. Cancer. 1976;37(3):1556-61.

5. Singh S, Lal P, Sikora SS, Datta NR. Squamous cell carcinoma arising from a congenital duplication cyst of the esophagus in a young adult. Dis Esophagus. 2001;14(3-4):258-61. https://doi.org/10.1046/j.1442-2050.2001.00204.x.

6. Wildi SM, Hoda RS, Fickling W, et al. Diagnosis of benign cysts of the mediastinum: the role and risks of EUS and FNA. Gastrointest Endosc. 2003;58(3):362-8. https://doi. org/10.1067/S0016-5107(03)00009-9.

7. Wiechowska-Kozłowska A, Wunsch E, Majewski M, Milkiewicz P. Esophageal duplication cysts: endosonographic findings in asymptomatic patients. World J Gastroenterol. 2012;18(11):1270-2. doi: 10.3748/wjg.v18. i11.1270.

8. Will U, Meyer F, Bosseckert H. Successful endoscopic treatment of an esophageal duplication cyst. Scand J Gastroenterol. 2005;40(8):995-9. https://doi. org/10.1080/00365520510023125.

9. Joyce AM, Zhang PJ, Kochman ML. Complete endoscopic resection of an esophageal duplication cyst (with video). Gastrointest Endosc. 2006;64(2):288-9. https://doi. org/10.1016/j.gie.2006.04.046.

10. Iyer A, Yadav S. Postoperative care and complications after thoracic surgery. En: Firstenberg MS (editor). Principles and practice of cardiothoracic surgery. Intech; 2013. p. 57-84. https://doi.org/10.5772/55351. 